\title{
Performance of Pancake Coils of Parallel Co-Wound Ag/BSCCO Tape Conductors in Static and Ramped Magnetic Fields*
}

\author{
S. W. Schwenterly, J. W. Lue, and M. S. Lubell \\ Oak Ridge National Laboratory ${ }^{* *}$, Oak Ridge, TN 37831-8040 \\ M. S. Walker, D. W. Hazelton, P. Haldar, J. A. Rice, J. G. Hoehn Jr., andd L. R. Motowidlo \\ Intermagnetics General Corporation, Latham, NY 12110-0460
}

\begin{abstract}
Critical Currents are reported for several Ag/BSCCO single-pancake colls in statlc magnetlc fields ranging from 0 to $5 T$ and temperatures from $4.2 \mathrm{~K}$ to $105 \mathrm{~K}$. The sample colls were co-wound of one to six tape conductors in parallel. Since the closed loops formed in such an arrangement could lead to eddy current heating or Instablilty in changing fields, one of the colls was also tested In hellum gas, In flelds ramped at rates of up to $1.5 \mathrm{~T} / \mathrm{s}$. For these quasi-adlabatic tests, at each temperature the transport current was set just below the critical value for a preset static field of 3.3 or $4.9 \mathrm{~T}$. The field was then rapidly ramped down to zero, held for $20 \mathrm{sec}$, and then ramped back up to the original value. The maximum observed temperature transient of about $1.7 \mathrm{~K}$ occurred at $9 \mathrm{~K}$, for a fleld change of $4.75 \mathrm{~T}$. The temperature transients became negligible when the sample was immersed in llquild hellum. Above $30 \mathrm{~K}$, the transients were below $1 \mathrm{~K}$. These results give confldence that parallel co-wound HTSC colls are stable in a rapidly-ramped magnetic field, without undue eddy current heating.
\end{abstract}

\section{INTRODUCTION}

Under an agreement between the Oak Ridge National Laboratory (ORNL) Superconductivity Partnership Center and Intermagnetics General Corporation (IGC), pancake coils of silver-sheathed $\mathrm{Bi}-2223 \mathrm{HTS}$ conductor are being developed and tested for electric power applications. The conductors are fabricated by the powder-in-tube (PIT) process. Useful current densities are now being achieved in conductor lengths over $100 \mathrm{~m}$, at temperatures and magnetic fields in the range of $20 \mathrm{~K}$ and $2 \mathrm{~T}$ or more [1], [2].

However, the final rolling step in the fabrication process reduces the conductor to a thin tape with a small cross section and relatively low tape operating current. High-field coils made with such a conductor would require many individuallyinsulated turns, which would reduce the overall winding current density and increase the inductance. If several tapes were wound in parallel with insulation only around the entire set, a more rugged conductor with higher total current, higher winding current density, and lower inductance could be made. Furthermore, a defect causing a small low-critical-current area in one tape could be bypassed by the others.

\footnotetext{
"Reaearch sponsored by the U.S. Department of Energy Advanced Utility Technology Concepts Division. Superconductivity Program for Electric Power Systems.

* Managed by Martin Marietta Energy Systems, Inc., for the U.S.D.O.E. under contract \#DE-AC05-840R21400.
}

A possible disadvantage of this approach is that eddy currents induced by rapidly-varying magnetic fields might produce high losses in the parallel tapes. Induced coupling currents might also produce flux-jump instabilities. This paper reports results of our investigations of the performance of pancake coils wound with multiple parallel silver-sheathed Bi-2223 conductors in rapidly-swept external magnetic fields.

\section{SAMPLES}

Each of the samples was a single pancake with an ID of $2.5 \mathrm{~cm}$. The approximate OD was $5 \mathrm{~cm}$ for Samples 1 and 2 and $6 \mathrm{~cm}$ for Samples 3 and 4. The conductor for each sample consisted of one or more PIT silver-sheathed tapes with a flat monocore of Bi-2223 HTS material. The Ag:SC ratio was 3:1 and the individual tape dimensions were $6 \mathrm{~mm} \times 0.1 \mathrm{~mm}$ for all samples. Voltage taps were installed only at the conductor ends. Parallel electrical connections between the tapes were also made only at the ends, though the parallel tapes touched all along their length. The pancakes were made with a windand-react process. Thus, there was no way to measure the individual tape critical currents before incorporating them into the sample. Table I gives other details on the samples.

\section{APPARATUS AND TECHNIQUE}

A commercial variable-temperature cryostat was used to maintain the samples at temperatures between $4.2 \mathrm{~K}$ and $100 \mathrm{~K}$ in 1-bar helium gas, by bleeding liquid helium out of an internal reservoir and heating it to the desired temperature. A copper cup surrounded the sample coil to help maintain an even temperature in the gas surrounding it $A$ heater on this cup provided further temperature control. External magnetic fields up to $5 \mathrm{~T}$ were provided by a water-cooled copper magnet surrounding the tail of the cryostat. The sample coil was mounted with its axis parallel to the extemal field. The field was thus parallel to the wide face of the conductor tape and to the $a-b$ plane of the HTS material for all tests.

Temperatures were measured by a silicon diode or ruthenium oxide thermometer mounted on the outer turns of

TABLE I

DESCRIPTIONOF SAMPLES

\begin{tabular}{lcccc}
\hline Sample & 1 & 2 & 3 & 4 \\
\hline No. of Tapes in Parallel & 1 & 6 & 2 & 2 \\
No. of Turns & 9 & 15 & 72 & 65 \\
Approximate OD $(\mathrm{cm})$ & 5 & 5 & 6 & 6 \\
Conductor Length(m) & 1.00 & 1.80 & 9.75 & 9.45 \\
\hline
\end{tabular}




\section{DISCLAIMER}

Portions of this document may be illegible in electronic image products. Images are produced from the best available original document. 
the pancake. Although a thermometer location inside the winding would have been preferable, we felt that this was not a great source of error as long as the temperature was changed slowly and the sample was kept in equilibrium with the surrounding gas. The single-pancake geometry of the sample exposes all turns to the gas and aids the equilibrium process. For the ramped-field experiments, the ac heating is presumably uniform in the conductor and thus not strongly dependent on thermometer location.

For the critical current measurements, the desired temperature was established and various constant fields were applied. The current was ramped up and down automatically and the sample $V-I$ curves were measured using the four-wire technique. Critical currents were determined using a $1-\mu \mathrm{V} / \mathrm{cm}$ criterion. No correction was made for current flow in the silver sheath at this gradient.

For the ramped-field experiments, the desired temperature was established and the extemal magnetic field was applied. The sample current was then set slightly below the critical value at these conditions. The field was ramped down to zero, held for about $20 \mathrm{sec}$, and ramped back to the original value. Since the silicon diode had a large magnetic field dependence at temperatures below $20 \mathrm{~K}$, a calibration curve had to be generated for the diode at each field used. The correction factor became quite large at the lowest temperatures. At $4.2 \mathrm{~K}$ and $4.9 \mathrm{~T}$, the indicated temperature was only $0.65 \mathrm{~K}$. This motivated the change to field-insensitive ruthenium oxide thermometers for Samples 3 and 4.

\section{RESULTS}

\section{A. Critical Currents}

Figs. 1-3 show critical currents $I_{C}$ vs field, for temperatures of 20,40 , and $77 \mathrm{~K}$. Since the samples had varying numbers of tapes in parallel, the current per tape is shown. This assumes that the current divides equally between tapes, although the individual tape $I_{C}$ 's were not measured. Sample 1, a single tape, and Sample 4, a double tape, showed the highest $I_{C}$ at $77 \mathrm{~K}$ and $40 \mathrm{~K}$ compared to the per-tape values of the others. For temperatures below $40 \mathrm{~K}$, the critical current is relatively insensitive to field, after an initial sharper drop at fields below $0.75 \mathrm{~T}$.

Samples 3 and 4 were investigated very thoroughly with respect to field and temperature. The qualitative behaviors of these two samples were similar, but critical currents for Sample 4 were a factor of 2.7 above those of Sample 3 at all temperatures and fields. Since both samples had the same conductor dimensions, this reflects a considerable improvement in current density for Sample 4. At fields below $0.75 \mathrm{~T}$, both samples showed a roughly linear dropoff of $I_{C}$ as the temperature was increased. However, at higher fields, $I_{c}$ tended to fall off below a linear dependence as the temperature fell below $20 \mathrm{~K}$. Fig. 4 shows this behavior in Sample 4.

Fig. 5 shows plots of the reduced critical current

$$
i_{C}(B)=I_{C}(B) / I_{C}(0)
$$

for Sample 4. For temperatures below $40 \mathrm{~K}$, the values of $i_{c}$ all fall close to a common curve. At higher temperatures, the

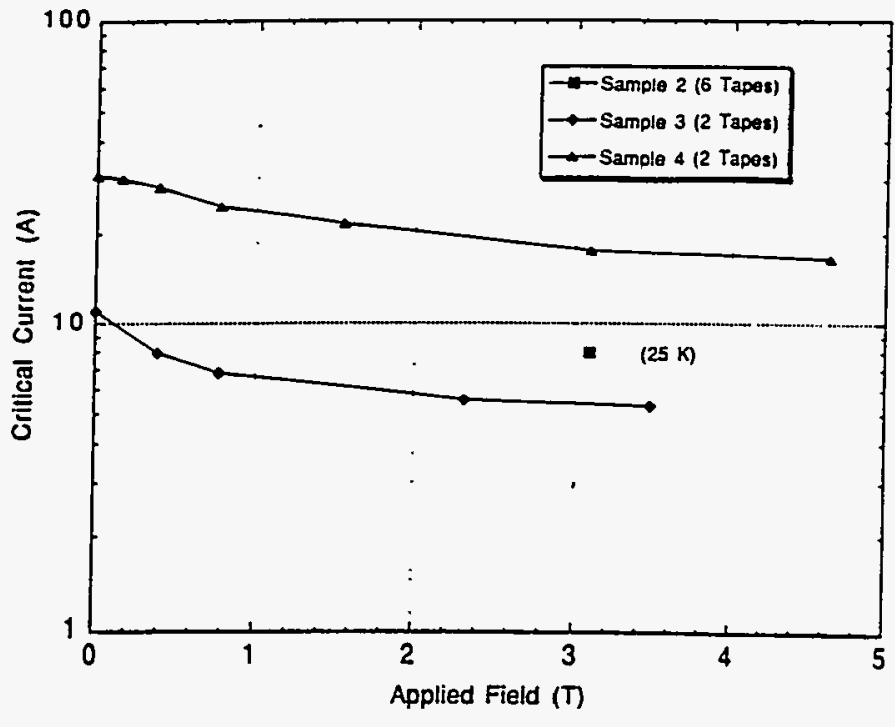

Fig. 1. Critical current vs applied field at $20 \mathrm{~K}$.

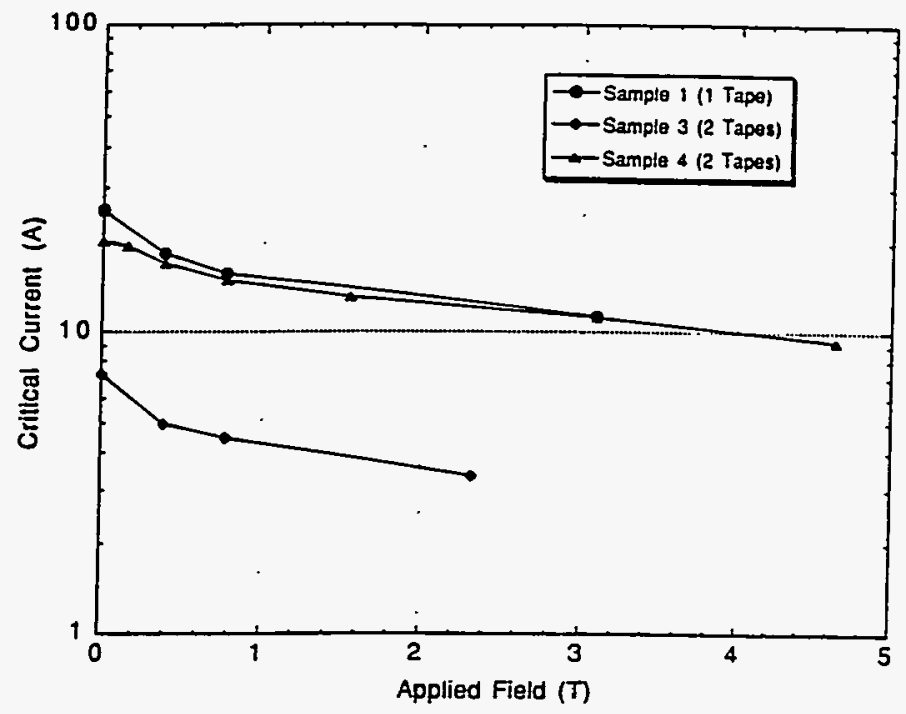

Fig. 2. Critical current vs applied field at $40 \mathrm{~K}$.

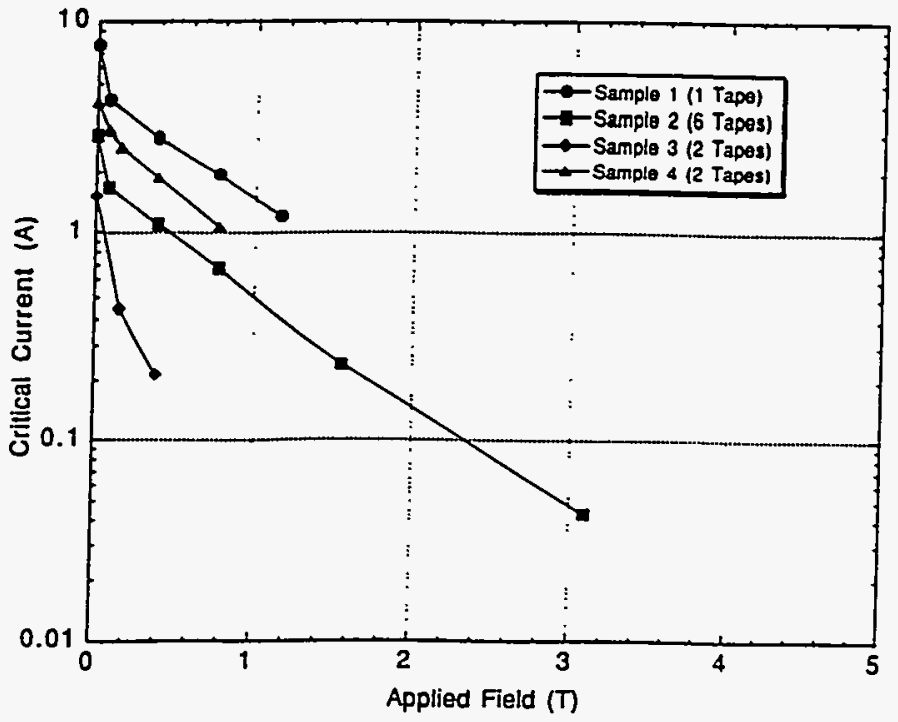

Fig. 3. Critical current vs applied field at $77 \mathrm{~K}$. 


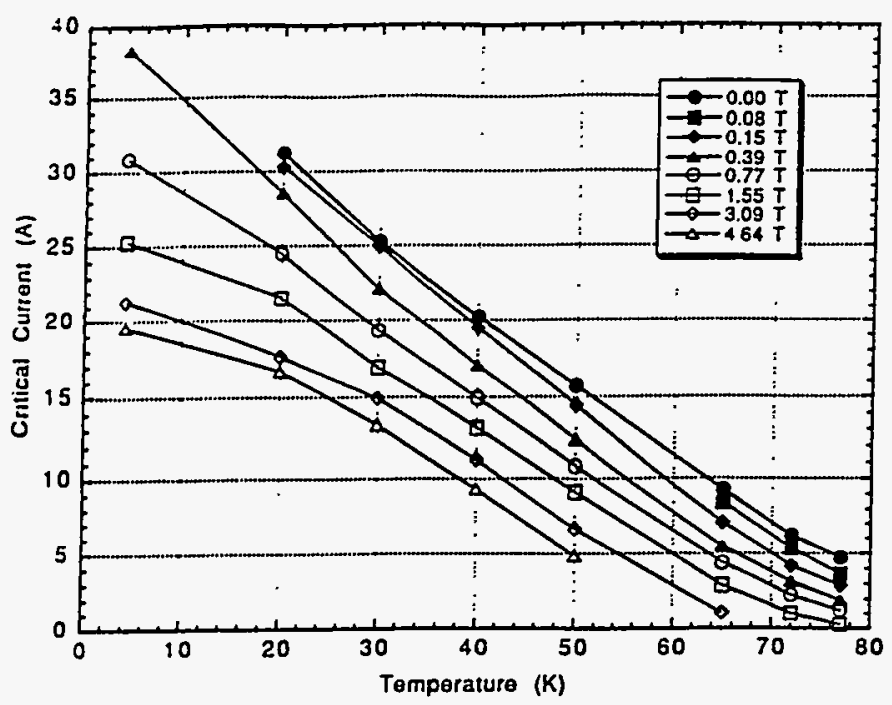

Fig. 4. Sample 4 critical current vs temperature and field.

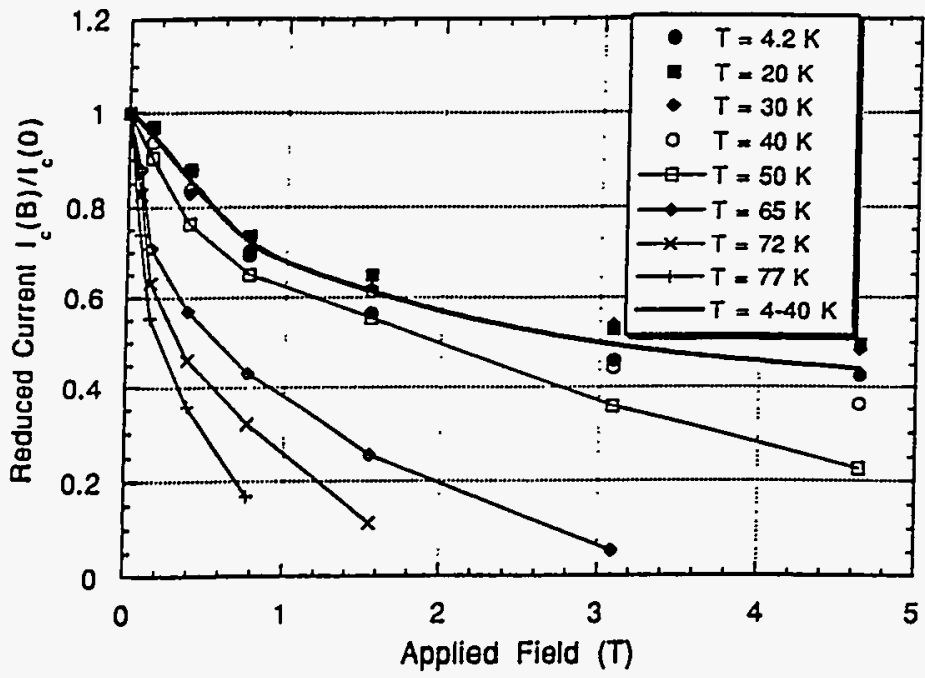

Fig. 5. Sample 4-reduced critical current vs field and temperanure.

$i_{c}$ curves drop off rapidly below this common curve, an indication of reduced pinning.

To investigate the sharpness of the superconductingnormal transition, the sample $V-I$ curves were fitted with the function

$$
V=k I^{n} \text {. }
$$

The $n$-value was generally in the neighborhood of 10 at low fields and low temperatures. At temperatures below 30 or $40 \mathrm{~K}$, it fell by roughly a factor of 2 as the field was raised above $1 \mathrm{~T}$, and was little affected by higher fields. At higher temperatures, $\boldsymbol{n}$ fell off much more rapidly with field, reaching values below 2 at $77 \mathrm{~K}$. These effects are illustrated by Fig. 6 , which shows plots of $n$ vs field at various temperatures for Sample 4.

A few tests were done on Sample 4 to check the history effect of increasing or decreasing magnetic field on the critical current. At $20 \mathrm{~K}$, observed $I_{C}$ 's were up to $8 \%$ higher in decreasing field than in increasing field. At $50 \mathrm{~K}$, this effect vanished.

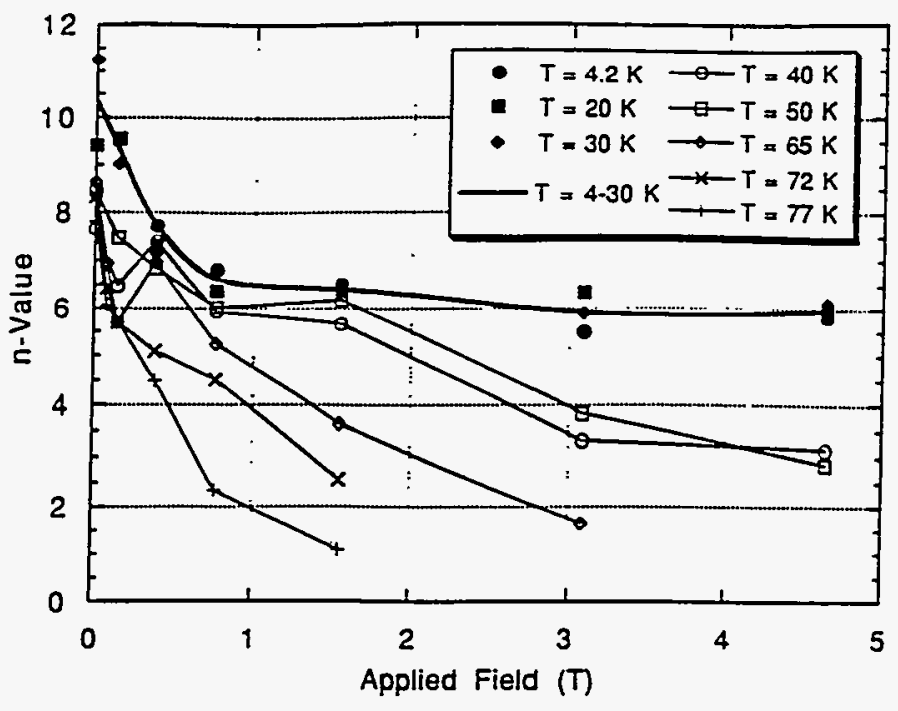

Fig. 6. Sample 4-n-value vs field and temperature.

\section{B. Ramped-Field Tests}

Most of the tests on Sample 2 were done in rapidlyramped magnetic fields. At temperatures below $30 \mathrm{~K}$, a small temperature rise could be observed due to the field change. Fig. 7 shows a typical plot of the field and field-corrected temperature vs time for a total field change of $4.75 \mathrm{~T}$, with a starting temperature of $7.4 \mathrm{~K}$. The dc transport current was set at $52 \mathrm{~A}$. The field fell from $4.9 \mathrm{~T}$ to $0.15 \mathrm{~T}$ during time 0 $10 \mathrm{sec}$ and ramped back up to $4.9 \mathrm{~T}$ during time $30-40 \mathrm{sec}$. The total temperature rise in this case is about $1.25 \mathrm{~K}$.

Fig. 8 shows the sample voltage and field vs time during the same field pulse shown in Fig. 7. Although large inductive voltage swings appear during the field ramps, the voltage returns nearly to zero in constant field, with no indication of a growing normal zone. A small resistive voltage due to the heating appears after the field pulse.

Fig. 9 shows the observed temperature rise with starting fields of $3.3 \mathrm{~T}$ and $4.9 \mathrm{~T}$, for sample temperatures between $4.5 \mathrm{~K}$ and $32 \mathrm{~K}$. No observable temperature rise occurred with

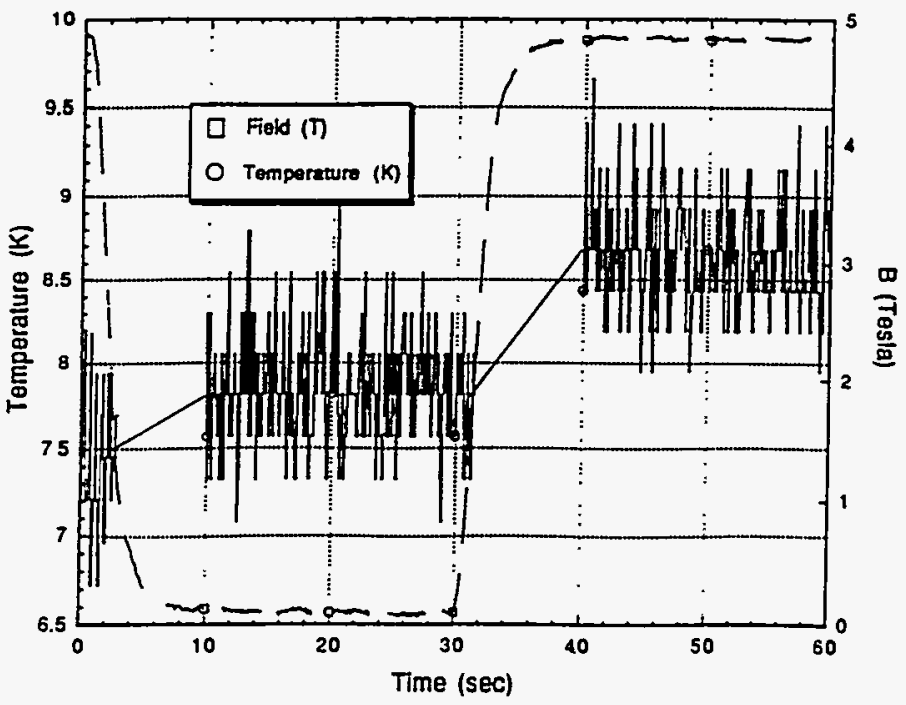

Fig. 7. Temperature and field vs time for Sample 2. 


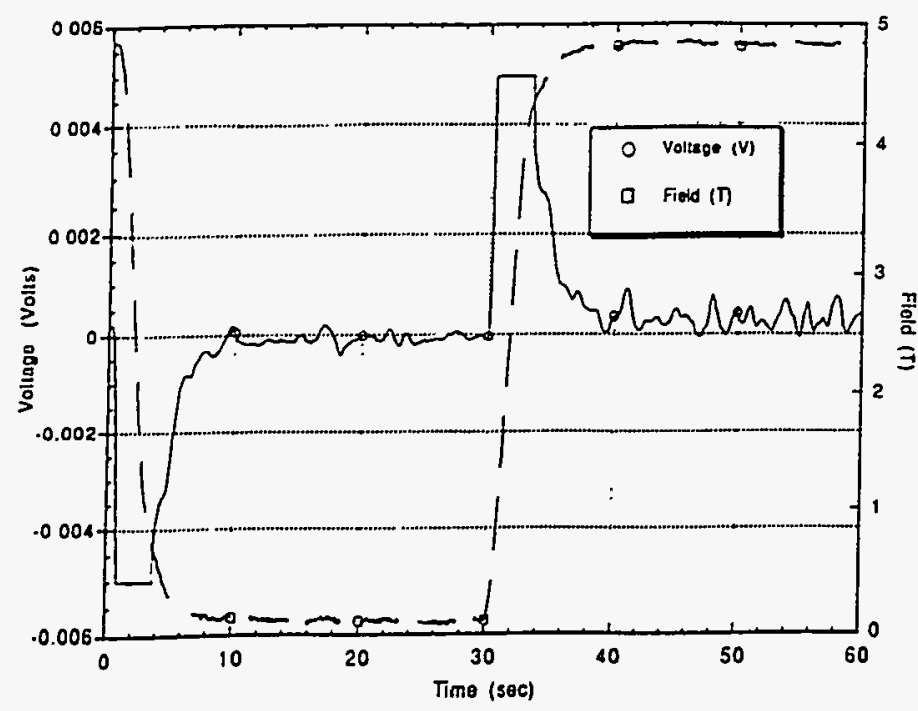

Fig. 8. Voltage and field vs time for Sample 2.

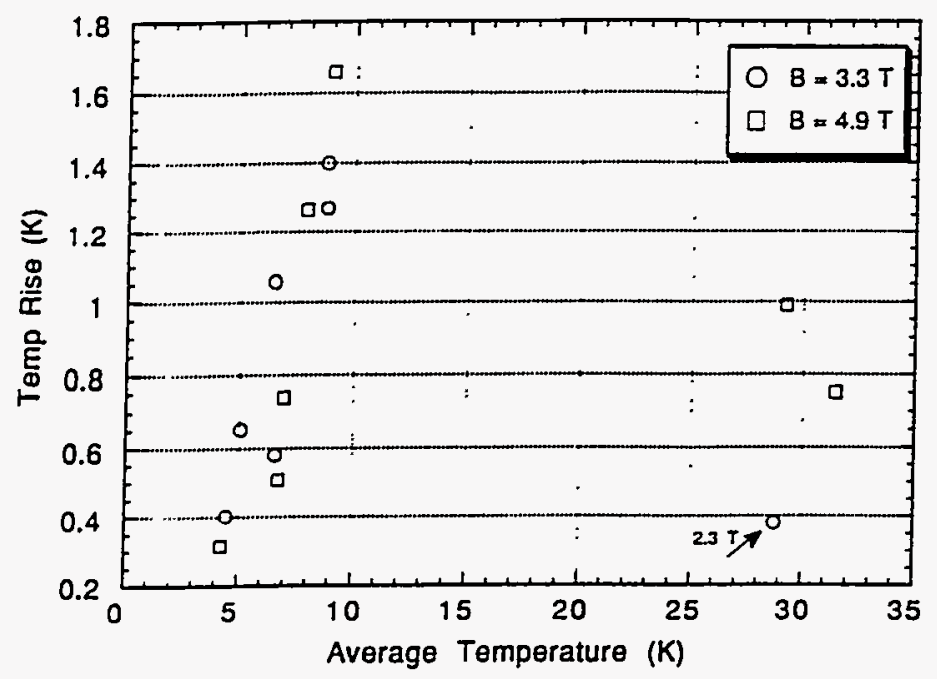

Fig. 9. Temperature rise vs average temperature for Sample 2.

the sample immersed in 4.2-K liquid helium, presumably due to the high heat transfer under these conditions. In helium vapor, the temperature rise increased rapidly as the temperature increased and the density and heat capacity of the surrounding gas fell. Surprisingly, no field dependence can be seen in the data between $4.2 \mathrm{~K}$ and $9 \mathrm{~K}$. Furthermore, the temperature rise is smaller during decreasing field than for increasing field. These features will be discussed in the next section. The highest observed temperature rise of about $1.7 \mathrm{~K}$ occurred with a 4.9-T starting field at $9 \mathrm{~K}$. There appears to be a peak in the temperature rise between $10 \mathrm{~K}$ and $20 \mathrm{~K}$. Unfortunately, time and funding did not allow further investigation of this range. At higher temperatures, the increasing heat capacity of the sample reduced the temperature rise, as shown by the 4.9-T points at $29 \mathrm{~K}$ and $32 \mathrm{~K}$.

\section{DISCUSSION}

The four samples have conductors with from one to six tapes in parallel. Examination of Figs. 1-3 shows that the critical current per tape in Sample 1 (1 tape) is the highest, followed by Sample 4 (2 tapes), Sample 2 (6 tapes), and Sample 3 (2 tapes). The per-tape currents for each sample are comparable to the short-sample currents of single tapes from the same batch (corrected for self-field), which indicates that winding and reacting multiple tapes in parallel does not lead to degraded individual tape performance. All the samples received essentially similar processing, and were made in the chronological order of their numbering scheme. The high performance of Sample 1 is due to the fact that it is a short length selected for good properties. The other samples have much longer tapes, which generally leads to lower performance due to processing difficulties and the greater chance of defects. The fact that Sample 4 could duplicate the performance of Sample 1 in nearly $20 \mathrm{~m}$ of tape shows the effects of ongoing improvements in processing technique.

For temperatures below $40 \mathrm{~K}$, the $n$-value and criticalcurrent data indicate much less sensitivity to magnetic field than at higher temperatures. Thus operating temperatures below $40 \mathrm{~K}$ are preferable for applications where the HTSC material must operate in substantial fields. Even at low temperatures, there is a considerable drop in $I_{C}$ as the field is raised from 0 to $0.75 \mathrm{~T}$. This is commonly attributed to the effect of grain boundaries. Since the objective of this work was to gather practical engineering data on the current-carrying capacity of the overall $\mathrm{Ag} / \mathrm{Bi}-2223$ composite, no corrections were made for current flow in the silver.

The ramped-field measurements can be compared to a model where eddy currents driven by the changing field are assumed to flow in the multiple-conductor sandwich and cooling by the surrounding helium is neglected. Dresner [3] has given formulas for the eddy current power per unit volume in plates of various shapes immersed in a uniform ramped magnetic field. The power is given by

$$
P=\frac{\dot{B}^{2} A f}{8 \pi \rho},
$$

where $\dot{B}$ is the field ramp rate, $A$ is the area presented by the plate normal to the field, $f$ is a geometric parameter, and $\rho$ is the plate resistivity. Since the field is uniform and the currents are constrained by the electrical insulation in the spiral path of the conductor, we can treat the conductor as if it were unrolled into a very long, thin rectangle of dimensions $2 a \times 2 b$.. In this case, the parameter $f$ is given by

$$
f=1.814\left(\frac{a}{b}+\frac{b}{a}\right)^{-1}
$$

For Sample 2, $a=0.9 \mathrm{~m}$ and $b=3 \times 10^{-4} \mathrm{~m}$, so that $f=6.05 \times 10^{-4}$. Iwasa et al [4] have measured the magnetoresistivity of silver at low temperatures. At $8 \mathrm{~K}$, the field dependence is quite linear and is given by

$$
\rho(B)=\left(2.0 \times 10^{-11}+2.8 \times 10^{-11} B\right) \Omega-\mathrm{m} .
$$


The temperature rise during a small time increment $d t$ is then

$$
d T=\left(P / C_{y}\right) d t,
$$

[5] L. Dresner, "Stability and protection of Ag/BSCCO magnets operated in the 20-40 K range," Cryogenics, vol. 33. pp. 900-909, 1993. where $C_{\nu}$ is the heat capacity per unit volume. Lowtemperature heat capacities for silver have been calculated by Dresner [5]. At $8 \mathrm{~K}$, the heat capacity is dominated by the phonon contribution and is about $1.1 \times 10^{4} \mathrm{~J}-\mathrm{m}^{-3}-\mathrm{K}^{-1}$. Numerical integration of these relations over the two 3-sec time periods in Fig. 7 where the field is rising and falling linearly gives a temperature rise of $0.35 \mathrm{~K}$ for falling field and $0.46 \mathrm{~K}$ for rising field. The value for rising field is higher, in agreement with Fig. 7, because the steepest part of the field ramp occurs at low field and low resistivity. The total calculated temperature rise is $0.81 \mathrm{~K}$, which is comparable to the observed value of $1.25 \mathrm{~K}$. The calculation assumes that the six tapes are stacked tightly together in the winding, whereas examination of the sample shows gaps between the tapes. Thus the effective area $A$ of the sample presented to the changing field is greater than the area of the tapes alone, which would increase the heating. Heating in the HTSC material could give another contribution. Eddy currents add to the dc transport current in half the tapes and subtract from it in the other half, with unclear overall effects on the sample heating. The lack of clear field dependence for the $4 \mathrm{~K}-9 \mathrm{~K}$ data in Fig. 9 is another area of disagreement with the eddy current model. Either the noise and magnetic field corrections for the thermometer have obscured the dependence, or else a more sophisticated model is necessary.

\section{CONCLUSION}

Critical current values have been reported for four samples of silver-sheathed Bi-2223 conductor. After an initial sharp drop at fields up to $0.75 \mathrm{~T}$, the critical current is relatively insensitive to field for temperatures below $40 \mathrm{~K}$. Performance drops off rapidly at higher temperatures. Winding several tapes in parallel basically multiplies the current capacity by the number of tapes. Operation of a multiple-tape sample in ramped magnetic fields does not cause excessive eddy current heating or instability.

\section{REFERENCES}

[1] M.P. Maley et al, "Anisotropy of transport properties normal and parallel to the tape plane in Bi-2223/Ag tapes," 1994 Applied Superconductivity Conference, Paper MEC-1, unpublished.

[2] M. Suenaga, P. Haldar, and L. Motowidlo, "In situ critical current measurements of $\mathrm{Bi}(2223: 2212) / \mathrm{Ag}$ composite tapes under tensile strain," 1994 Applied Superconductivity Conference, Paper MEC-8, unpublished.

[3] L. Dresner, "Eddy current heating of irregularly-shaped plates by slow ramped fields," Proc. 8th Symp. on Eng. Probs. of Fusion Research, p.89, 1979 [IEEE Publ. No. 79 . CH1-441-5-NPS 89].

[4] Y. Iwasa et al, "Magnetoresistivity of silver over the temperafure range $4.2-159 \mathrm{~K}$," Cryogenics, vol. 33, pp. 836-837, 1993.

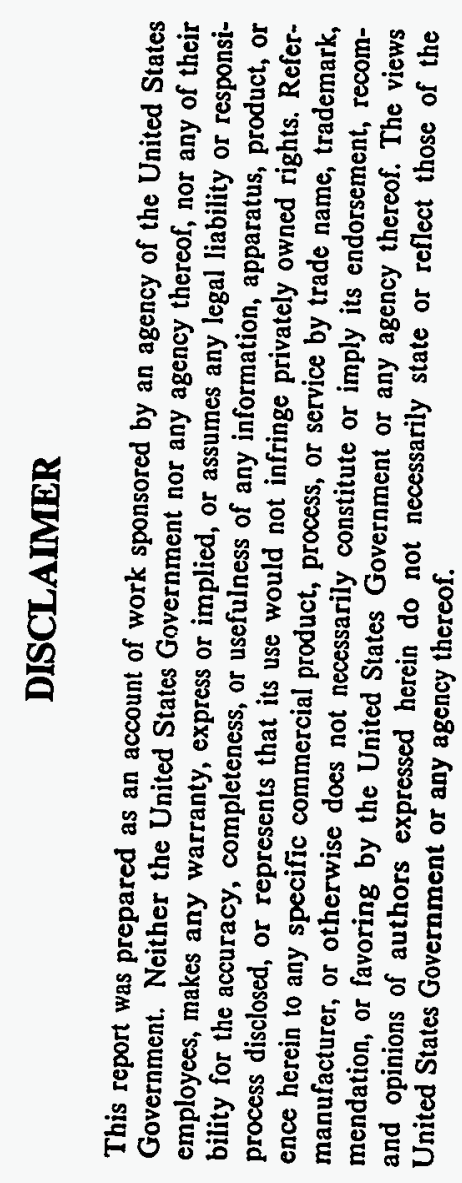

